\title{
A PRELIMINARY REPORT OF A NEW GELATIN LIQUEFACTION METHOD
}

\author{
BY \\ J. KOHN \\ From Queen Mary's Hospital, Roehampton, London
}

(RECEIVED FOR PUbliCATION MARCH 30, 1953)

This method depends upon the use of formalindenatured gelatin with finely powdered charcoal incorporated as an indicator of liquefaction. The gelatin charcoal is used only as a substrate for testing the gelatinase activity and not as a nutrient medium. This substrate contains a large number of minute particles of charcoal with a comparatively small amount of gelatin as binding substance. Consequently even small quantities of liquefied gelatin will release appreciable numbers of easily recognized charcoal particles.

Denatured gelatin does not melt, hence it can be sterilized and incubated at any desired temperature. In other respects it behaves like natural gelatin.

The medium is prepared from Difco nutrient gelatin which is mixed with tap water in the proportion of $15 \mathrm{~g}$. to $100 \mathrm{ml}$. of water. To the melted gelatin finely powdered charcoal is added in a proportion of 3 to $5 \mathrm{~g}$. per $100 \mathrm{ml}$. of the gelatin medium. The mixture is thoroughly shaken, and poured into Petri dishes or other suitable flat containers to form a layer about $3 \mathrm{~mm}$. thick. The mixture should be poured when quite cool so that it sets quickly before the charcoal can sediment. It is advisable to smear the bottom of the container very thinly with vaseline, as the charcoal gelatin mixture has a tendency to stick to glass.

After the mixture has thoroughly set the whole sheet is lifted from the dish and placed in $10 \%$ formalin for 24 hours. The formalized gelatin sheet is then punched into discs about $1 \mathrm{~cm}$. diameter or cut into strips of about $2 \mathrm{~cm}$. length and 5 to $8 \mathrm{~mm}$. width. The pieces of gelatin are then wrapped in gauze and placed in a basin under running tap water for 24 hours. The material is then ready for sterilization.

The pieces of prepared gelatin are best put into screw-capped bottles and covered with water. The bottles are then placed in a water-bath for tyndallization or into a steamer. Sterilization is effected by steaming for 30 minutes or by repeated heating in a water-bath at about 90 to $100^{\circ} \mathrm{C}$. for 20 minutes each time. Autoclaving is not recommended, as it tends to soften the gelatin. After sterilization the water is decanted from the containers and the material is ready for use. The gelatin pieces can now be put under sterile conditions into any suitable fluid medium and incubated to check their sterility. The prepared media are then ready for use.

The test proper is performed by inoculating the medium in which the gelatin is suspended and incubating it at $37^{\circ} \mathrm{C}$. The test can also be performed on solid media, e.g., nutrient agar, in which case the agar slope is inoculated first and a sterile gelatin disc or strip is placed on the surface of the agar, preferably near the bottom of the slope. Care should be taken that a certain amount of condensation water is present. An already established culture, whether in a fluid or on a solid medium, can be tested in the same way as described above. There may even be some advantage in doing so, as a certain amount of gelatinases may be present in the culture to start with.

The first evidence of liquefaction is the appearance of free particles of charcoal sedimenting to the bottom of the medium. On shaking they become resuspended and form a clearly visible black cloud. Once the process has started it proceeds rapidly to total liquefaction. Mechanical factors such as shaking do not produce false positive results.

The method has been tested with various organisms, mainly with slow liquefiers of the Bacteriaceae family and also with anaerobes of the Clostridia group and has given very satisfactory and consistent results. Some strains of the $B$. cloacae aerogenes group which required seven days to produce definite liquefaction by the standard method (gelatin stab at $22^{\circ} \mathrm{C}$ ) have given unequivocal results within 24 hours. On an average the time required to obtain definite evidence of liquefaction was much shorter than that required by the standard method. False negative results have not so far been encountered.

A fuller report on the application of this method is being prepared. 